\title{
Reaction Mechanism and Kinetics of the Traceless Staudinger Ligation
}

\author{
Matthew B. Soellner, Bradley L. Nilsson, and Ronald T. Raines*
}

Contribution from the Departments of Chemistry and Biochemistry,

University of Wisconsin-Madison, Madison, WI 53706

E-mail: raines@biochem.wisc.edu

\begin{tabular}{|c|c|}
\hline Page & Contents \\
\hline S1 & Table of Contents \\
\hline S2 & Figure S1 (phosphinothiophenol 23) \\
\hline S3 & Figure S2 (phosphinoalcohol 24) \\
\hline S4 & Figure S3 (phosphinothiol 25) \\
\hline S5 & Figure S4 (phosphinophenol 26) \\
\hline
\end{tabular}



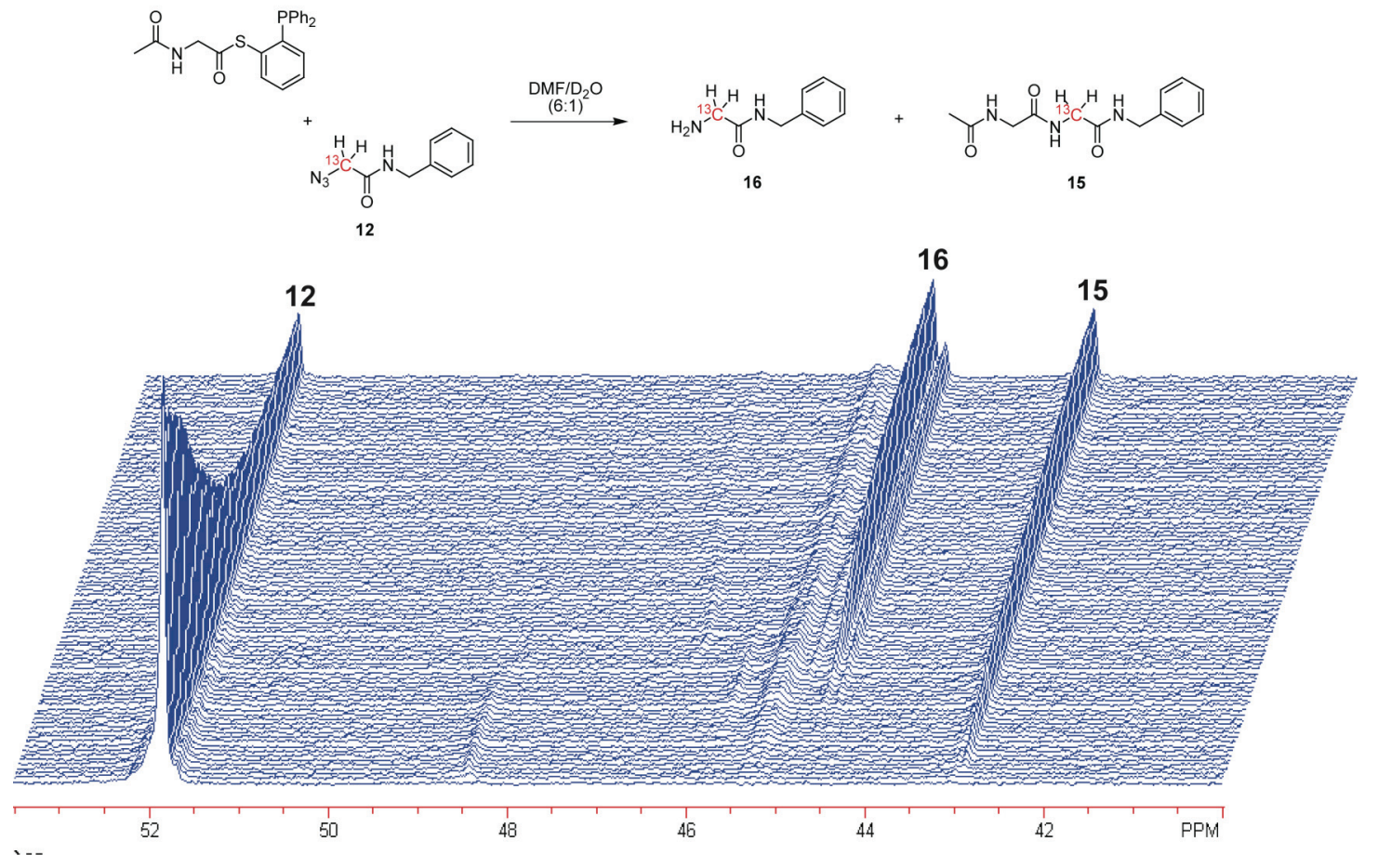

Figure S1. ${ }^{13} \mathrm{C}$ NMR Assay of a Traceless Staudinger Ligation mediated by phosphinothiophenol 23. The reaction was performed at room temperature with reagent concentrations of $0.175 \mathrm{M}$. Ninety spectra were acquired over a 12-h time course, scaled to enable the calculation of the second-order rate constant and reaction yield (Table 2). Peak integrations were calibrated with a standard curve generated with purified ${ }^{13} \mathrm{C}$-labeled species. 


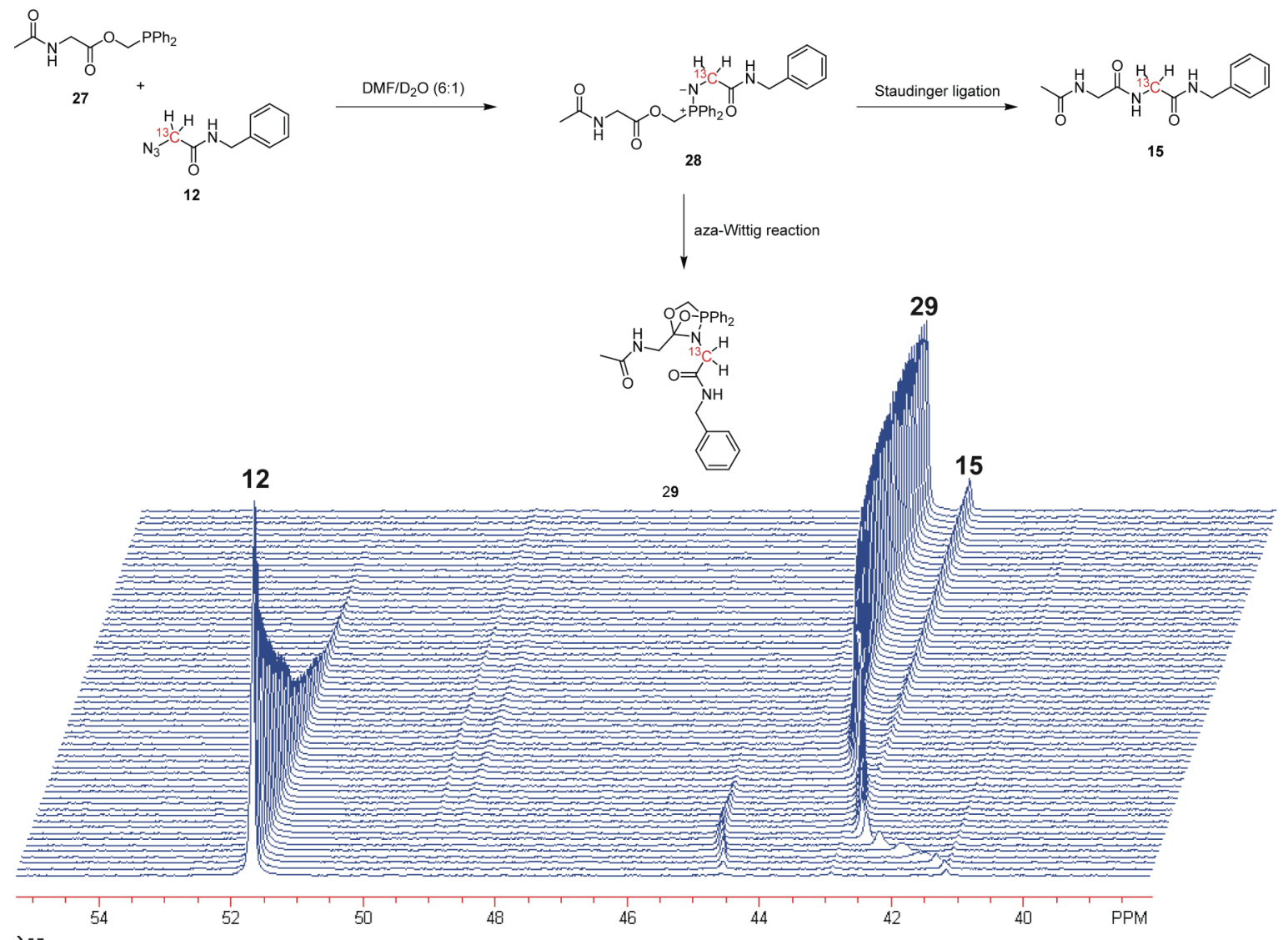

Figure S2. ${ }^{13} \mathrm{C}$ NMR Assay of a Traceless Staudinger Ligation mediated by phosphinoalcohol 24. The reaction was performed at room temperature with reagent concentrations of $0.175 \mathrm{M}$. Ninety spectra were acquired over a 12-h time course, scaled to enable the calculation of the secondorder rate constant and reaction yield (Table 2). Peak integrations were calibrated with a standard curve generated with purified ${ }^{13} \mathrm{C}$-labeled species. 


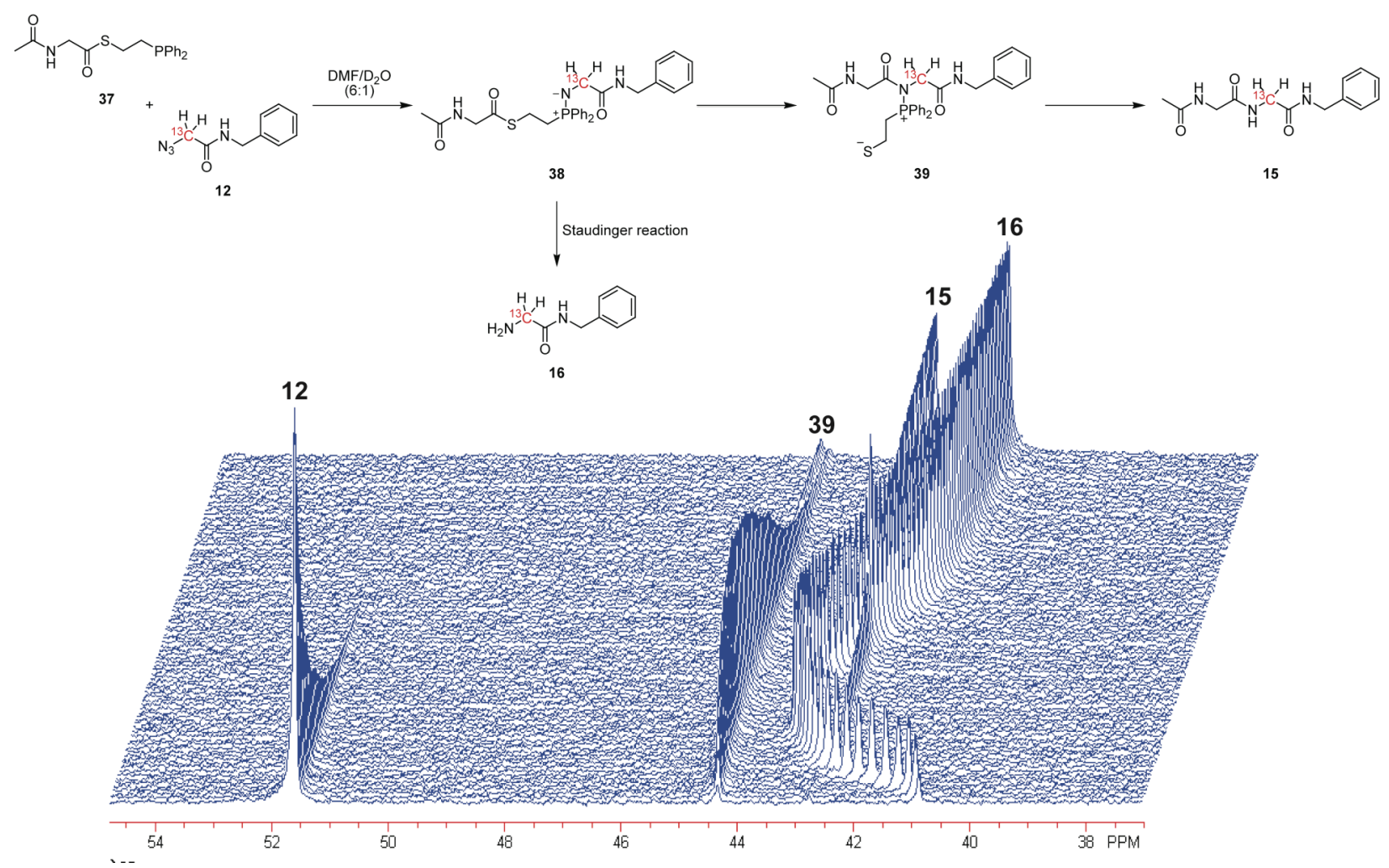

Figure S3. ${ }^{13} \mathrm{C}$ NMR Assay of a Traceless Staudinger Ligation mediated by phosphinothiol 25. The reaction was performed at room temperature with reagent concentrations of $0.175 \mathrm{M}$. Ninety spectra were acquired over a 12-h time course, scaled to enable the calculation of the second-order rate constant and reaction yield (Table 2). Peak integrations were calibrated with a standard curve generated with purified ${ }^{13} \mathrm{C}$-labeled species. 


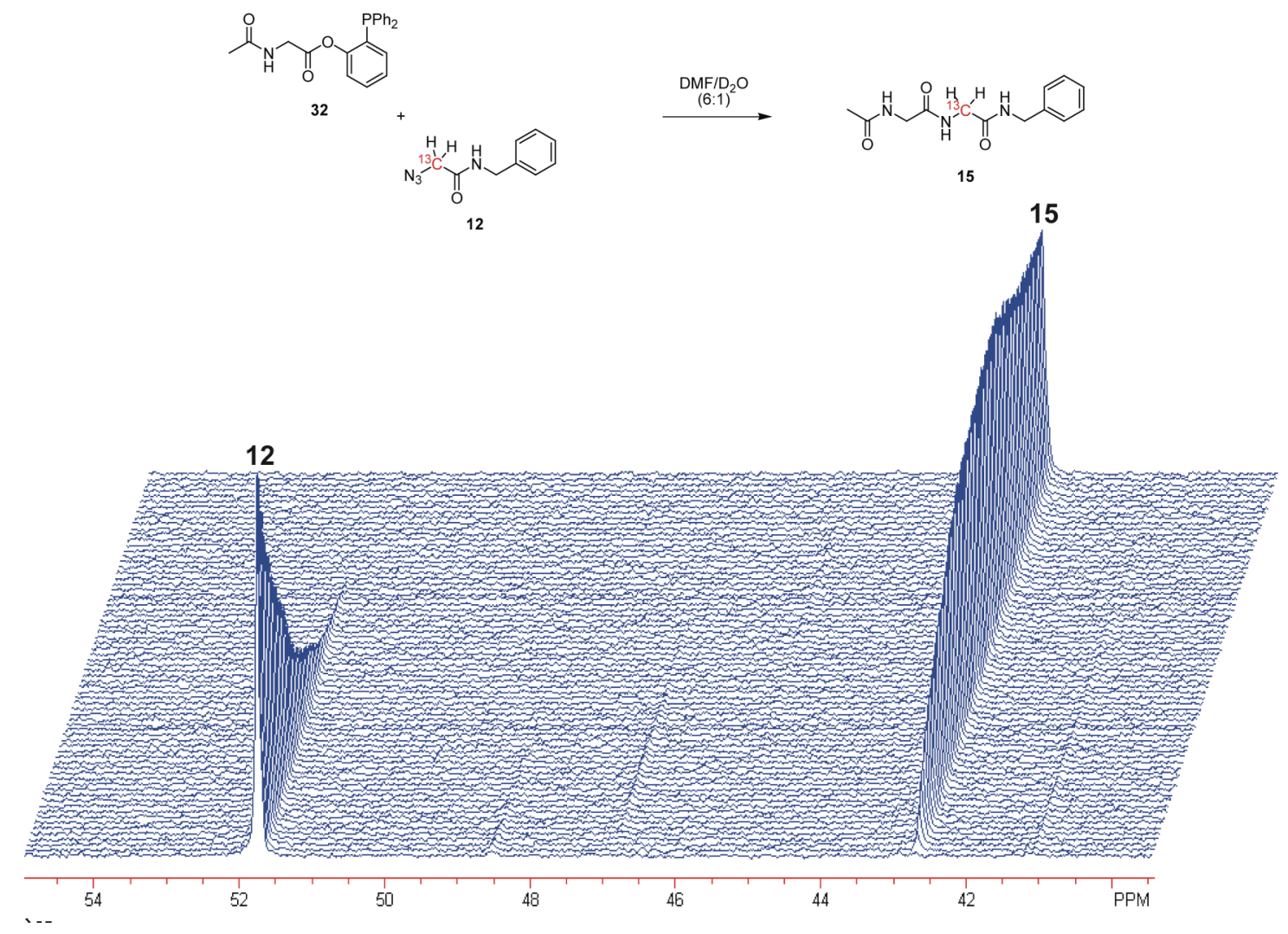

Figure S4. ${ }^{13} \mathrm{C}$ NMR Assay of a Traceless Staudinger Ligation mediated by phosphinophenol 26. The reaction was performed at room temperature with reagent concentrations of $0.175 \mathrm{M}$. Ninety spectra were acquired over a 12-h time course, scaled to enable the calculation of the second-order rate constant and reaction yield (Table 2). Peak integrations were calibrated with a standard curve generated with purified ${ }^{13} \mathrm{C}$-labeled species. 